\title{
Adaptation and validation of the Patient Expectations and Satisfaction with Prenatal Care instrument among Brazilian pregnant women ${ }^{1}$
}

\author{
Patrícia Santos Prudêncio \\ Fabiana Villela Mamede ${ }^{3}$ \\ Rosana Aparecida Spadoti Dantas ${ }^{4}$ \\ Luiz de Souza $^{5}$ \\ Marli Villela Mamede ${ }^{6}$
}

\begin{abstract}
Objective: to adapt and validate the Patient Expectations and Satisfaction with Prenatal Care instrument for use in Brazil. It contains 41 items divided into two dimensions: expectations and satisfaction. The adapted version was submitted to analysis for stability, convergent construct validity, and internal consistency (Cronbach's alpha) for distinct groups and dimensions. Method: 119 pregnant women receiving prenatal care were interviewed and 26 of these women answered the instrument twice (retest). Internal consistency was appropriate (Cronbach's alpha $\geq 0.70$ ); test-retest presented strong correlation $(r=0.82 ; p<0.001)$ for the domain expectations and moderate correlation $(r=0.66 ; p<0.001)$ for the satisfaction domain. The analysis confirmed that the instrument's adapted version is valid in the studied group. Results: there is strong evidence for the validity and reliability of the instrument's adaptation. Conclusion: the instrument needs to be tested in groups of pregnant women with different social characteristics.
\end{abstract}

Descriptors: Validation Studies; Patient Satisfaction; Prenatal Care.

\footnotetext{
${ }^{1}$ Paper extracted from master's thesis "Adaptação cultural e validação para o uso no Brasil do instrumento: Patient Expectations and Satisfaction with Prenatal Care (PESPC)" presented to Escola de Enfermagem de Ribeirão Preto, Universidade de São Paulo, WHO Collaborating Centre for Nursing Research Development, Ribeirão Preto, SP, Brazil.

2 Master's Student, Escola de Enfermagem de Ribeirão Preto, Universidade de São Paulo, WHO Collaborating Centre for Nursing Research Development, Ribeirão Preto, SP, Brazil.

3 PhD, Professor, Escola de Enfermagem de Ribeirão Preto, Universidade de São Paulo, WHO Collaborating Centre for Nursing Research Development, Ribeirão Preto, SP, Brazil.

${ }^{4}$ PhD, Associate Professor, Escola de Enfermagem de Ribeirão Preto, Universidade de São Paulo, WHO Collaborating Centre for Nursing Research Development, Ribeirão Preto, SP, Brazil.

${ }^{5} \mathrm{PhD}$, Professor, Faculdade de Medicina de Ribeirão Preto, Universidade de São Paulo, Ribeirão Preto, SP, Brazil.

${ }^{6}$ PhD, Full Professor, Escola de Enfermagem de Ribeirão Preto, Universidade de São Paulo, WHO Collaborating Centre for Nursing Research Development, Ribeirão Preto, SP, Brazil.
} 


\section{Introduction}

The definition of patient satisfaction is considered to be a multidimensional construct ${ }^{(1-3)}$ that includes the perceptions, attitudes and expectations ${ }^{(6)}$ patients hold in relation to healthcare ${ }^{(4-5)}$.

Satisfaction is influenced by the expectations an individual has concerning the type, content and quality of care received(3). Patient satisfaction can be seen as an attitude, that is, a general orientation of an individual toward an entire experience with healthcare. It can be understood as having cognitive and emotional facets, which are related to expectations, previous experiences and social networks ${ }^{(7)}$.

Some authors argue that patient satisfaction (attitudes in relation to one's experience with healthcare) is determined by the interaction between one's expectations (beliefs concerning healthcare) and characteristics of the healthcare received ${ }^{(7-8)}$. When including mechanisms to assess patient satisfaction, one should take into account the ability of users to understand what they are being asked and communicate their opinions and feelings efficiently. It is known that levels of literacy (intellectual), levels of physical/sensorial impairment, poor language proficiency, and ethnic and cultural diversity are factors that may influence the assessment of satisfaction. Similarly, social factors such as socioeconomic status, demographic characteristics (urban/rural) and technology should be considered, especially when these signal whether the consumer will provide feedback and express his/her satisfaction, or lack of satisfaction, with the service provided ${ }^{(7)}$.

It is important to assess the satisfaction of pregnant women with prenatal care because the results of one's dissatisfaction with prenatal care can be disastrous, resulting in the patient abandoning treatment or reducing attendance to the health service. Thus, health care delivery may become less efficient, and worsen birth outcomes ${ }^{(9)}$.

In Brazil, research addressing the expectations and satisfaction of pregnant women in relation to prenatal care is still incipient. One of the difficulties assessing the expectations and satisfaction of pregnant women in relation to prenatal care is a lack of valid and reliable instruments, in addition to methodological flaws in some existing measurement instruments ${ }^{(9)}$. Even though some instruments assessing the satisfaction of women with prenatal care were found in the literature, there are no instruments adapted and/or validated for Brazil. Hence, this study's objectives were to culturally adapt and analyze the psychometric properties of the instrument Patient Expectations and Satisfaction with Prenatal Care $(P E S P C)^{(9)}$.

\section{Method}

This methodological study of the cultural adaptation and validation of the PESPC ${ }^{(9)}$ instrument was conducted according to the stages recommended in Brazilian and international literature, described as follows.

The study was initiated after the authors of the instrument's original version authorized it and approval from the City Health Department and the Institutional Review Board at the University of São Paulo at Ribeirão Preto, College of Nursing (No. 1250/2010) was obtained. All the pregnant women expressed their consent to participate in the study by signing free and informed consent forms.

For cultural adaptation and validation, the original instrument was submitted to an Expert Committee to perform face and content validity tests. Ten pregnant women undergoing prenatal care provided the instrument's semantic analysis. The PESPC's adapted version was pretested with a sample of 40 pregnant women who did not report any problems understanding or responding to the instrument. The adapted version was tested with 119 pregnant women undergoing prenatal care in one of the three units selected in the city of Ribeirão Preto, SP, Brazil: Women's Health Referral Unit (Mater), Family Health Family (Maria Casagrande), and the Gynecological and Obstetrical Outpatient Clinic of the Health Unit at the University of São Paulo at Ribeirão Preto, Medical School (CSE-FMRP-USP). The participants were randomly approached while waiting for their prenatal consultations in one of these three units. Twenty-six, out of the initial 119 participants, completed the PESPC's adapted version a second time 14 days after the first assessment.

Inclusion criteria were: being pregnant and 18 years old or older; receiving prenatal care in one of the selected units; having attended at least two prenatal care consultations; having the cognitive capacity to answer the instrument's questions.

\section{Data collection}

Information concerning the socio-demographic and clinical-obstetrical characterization of this study's participants was collected during individual interviews and through consultation of their medical files. 
PESPC was originally developed in the United States of America $^{(9)}$ and later used by other researchers(10-11). It is composed of 41 items, divided into two domains: expectations and satisfaction. Each domain contains four subscales. The subscales in the expectation domain are: Complete Care, Provider Continuity, Personalized Care, and Other Services. The subscales in the satisfaction domain include: Information, Provider Care, Staff Interest, and System Characteristics. A Likert scale ranging from (1) totally agree to (6) totally disagree is used. Lower scores refer to higher levels of expectations and satisfaction and higher scores refer to lower levels of expectations and satisfaction. The interval for the expectations domain ranges from 12 to 72 and from 29 to 174 for the satisfaction domain. The intervals for each subscale are: complete care (from 4 to 24); provider continuity (from 2 to 12 ); personalized care (from 4 to 24); other services (from 2 to 12); information (from 7 to 42 ); provider care (from 6 to 36); staff interest (from 6 to 36); and system characteristics (from 10 to $60)$. The internal consistency obtained on the original version for the instrument's domains was appropriate ( $\alpha=0.72$ for the expectations domain and $\alpha=0.94$ for the satisfaction domain $)^{(9)}$.

The Brazilian version of the Patient Satisfaction Instrument (PSI) ${ }^{(12)}$ was used to test convergent construct validity. The PSI was developed by Hinshaw and Atwood ${ }^{(13)}$ to assess patient satisfaction in relation to nursing care. It is composed of 25 items encompassing three dimensions or components: technical-professional care (7 items), trust (11 items), and patient education (7 items). The answers to each domain are placed on a Likert scale that ranges from (1) strong agreement to (5) strong disagreement; lower scores indicate higher levels of satisfaction. The PSI has been used to assess different aspects involving nursing care ${ }^{(12)}$. In this study, only the trust domain was used to test convergent construct validity.

\section{The PESPC's adaptation and validation process}

All the stages recommended in the literature were employed during the instrument's cultural adaptation ${ }^{(14)}$ : initial translation, synthesis of translations, an expert committee, back translations, semantic validation and pretest. The PESPC's original version was submitted to face and content validation by an Expert Committee and to semantic validation by ten pregnant women undergoing prenatal care. The Expert Committee, composed of seven nurses (all experts in the women's health field, and one expert in the method used), was asked to assess the PESPC's adapted version in terms of cultural, semantic, idiomatic and conceptual equivalence. The instrument was semantically adapted from English to Brazilian Portuguese and the necessary adjustments were made for the version to be used in Brazil. The version obtained after changes were suggested by the committee was submitted to semantic analysis to verify whether all the items in the scale were comprehensible by the study's target population(15).

The participants were asked about each item's comprehensibility. They reported some difficulties and made some suggestions for the items to be more easily understood by other pregnant women. Some of the suggestions were accepted, thus some terms were replaced in some of the instrument's items. Examples of such changes include: "convenience" was replaced by "desire"; "express" by "show"; "partially" by "somewhat". Such changes were intended to improve understanding of the items contained in the adapted version by pregnant women undergoing prenatal care in Brazil.

The pretest was initiated after this stage. Interviews were held with 40 patients. The instrument's reliability was tested through internal consistency (Cronbach's alpha): 0.82 for the expectations domain and 0.95 for the satisfaction domain. No comprehension problems were reported by the women participating in this stage, thus, the version was not changed. Afterwards, the following psychometric properties were verified: (convergent and divergent) construct validation, reliability in terms of internal consistency (Cronbach's alpha), and measurement stability (test-retest). A sample of 119 pregnant women undergoing prenatal care was selected for this stage. They were randomly chosen while waiting for prenatal consultations. It is worth noting that some researchers analyzed different sample sizes and observed that a sample between 50 and 100 individuals is appropriate for obtaining results to demonstrate an instrument's validity ${ }^{(16)}$.

Twenty-six out of the 119 pregnant women who participated in the first assessment of the PESPC's adapted version were randomly selected for the measurement's stability verification. This small group was invited to participate in the second assessment of the PESPC's adapted version at an interval of 10 to 14 days between the interviews (test-retest). This period of time was chosen taking into account that the time should be long enough for women to be unable to recall the answers from the first assessment but short enough to ensure no clinical change would occur(17); the length 
of this interval was chosen based on recommendations found in the literature ${ }^{(18)}$.

Convergent construct validity was verified by comparing the PESPC's adapted version with the measure of satisfaction obtained by the PSI. The adapted instrument's divergent (discriminant) construct validity was verified by comparing the groups in terms of gestation period (first, second or third trimester), marital status (married, single, or cohabitating), number of pregnancies (one, two or multiple pregnancies), occupation (paid job or homemaker), religion (Catholic, Evangelical, or other), educational level (incomplete primary/middle school, complete primary/middle school, complete high-school or college degree), monthly family income (less than one time the minimum wage, 1 to 2 times, 2 to 3 times, or 3 times or more). Factor analysis with Varimax rotation was performed to assess dimension-related construct validity.

\section{Statistical analysis}

The variables were coded and organized in a Microsoft Office Excel database. The statistical program STATA, version 10, was used for data analysis. Descriptive analysis was performed for all the variables. Central tendency (average and median) and dispersion (standard deviation) measures were computed for the continuous variables. Internal consistency was estimated using Cronbach's alpha; results that reached from 0.70 to 0.90 were considered to be appropriate ${ }^{(19)}$. Stability was measured by test-retest. Hypotheses were established regarding the relationship between the measures of satisfaction obtained with the PESPC's adapted version and the measures of satisfaction obtained with the PSI for the analysis of convergent construct validity through computation of Pearson's linear correlation coefficient. Divergent construct analysis between groups was performed by computing the $\mathrm{p}$-value obtained using the ANOVA test. The dimension-related construct validity of the PESPC's adapted version was verified through factor analysis with Varimax rotation. The level of significance was fixed at 0.05 .

\section{Results}

A total of 119 pregnant women cared for in the three public health services selected in the city of Ribeirão Preto, SP, Brazil participated in the study. The average age was 25 years old $(S D=5.3) ; 48(40.3 \%)$ were married; 51 (42.9\%) were primiparas; 76 (63.9\%) were homemakers; 69 (57.9\%) completed high school and only four (3.3\%) had a college degree.

In regard to the total descriptive analysis, for each of the scale's domains (expectations and satisfaction) and each item, an average value of 9.0 (4.7) was obtained for the expectations domain and 17.8 (7.2) for the satisfaction domain. The average value for the expectations domain's items was between 5.2 and 14.4 and between 17.8 and 29.2 for the satisfaction domain's items. The intervals for each domain (expectations and satisfaction) were 13 to 62 and 31 to 143 , respectively (Table 1).

Table 1 - Descriptive statistics concerning the results obtained in the subscales of the adapted version of the Patient Expectations and Satisfaction with Prenatal Care (PESPC) instrument ( $n=119)$. Ribeirão Preto, SP, Brazil 2012

\begin{tabular}{|c|c|c|c|c|c|}
\hline Subscales & No. of items & Possible interval & Interval obtained & Median & Average (SD) \\
\hline Expectations & 12 & $12-72$ & $13-62$ & 7.5 & $9.0(4.7)$ \\
\hline Complete care & 4 & $4-24$ & $4-22$ & 14 & $14.4(4.4)$ \\
\hline Provider continuity & 2 & $2-12$ & $2-10$ & 4 & $5.0(2.0)$ \\
\hline Personalized care & 4 & $4-24$ & $5-20$ & 11 & $11.5(3.6)$ \\
\hline Other services & 2 & $2-12$ & $2-10$ & 4 & $5.2(2.0)$ \\
\hline Satisfaction & 29 & $29-174$ & $31-143$ & 17.5 & $17.8(7.2)$ \\
\hline Information & 7 & $7-42$ & $8-31$ & 17 & $17.8(4.9)$ \\
\hline Provider care & 6 & $6-36$ & $7-33$ & 12 & $13.4(3.8)$ \\
\hline Staff interest & 6 & $6-36$ & $6-30$ & 13 & $15.1(4.9)$ \\
\hline System characteristics & 10 & $10-60$ & $10-49$ & 28 & $29.2(7.8)$ \\
\hline
\end{tabular}

The results found in the analysis of psychometric properties concerning divergent construct validity investigated among the studied groups reveal the following variables to be statistically significant in the expectations domain: number of children $(p<0.001)$, occupation $(p=0.0042)$ and education $(p=0.052)$. The following variables were statistically significant in the satisfaction domain: gestation period $(p=0.034)$ and marital status $(p=0.043)$. These results are presented in Table 2. 
Table 2 - Comparison of averages (analysis of variance) of the sum of scores concerning the expectations and satisfaction domains according to gestation period, marital status, number of children, occupation, religion, educational level, monthly family income $(n=119)$. Ribeirão Preto, SP, Brazil 2012

\begin{tabular}{lcc}
\hline \multicolumn{1}{c}{ Variable } & Expectations* $^{*}$ & Satisfaction* $^{*}$ \\
\hline Gestation period & 0.977 & 0.034 \\
Marital status & 0.341 & 0.043 \\
Number of children & 0.001 & 0.719 \\
Occupation & 0.042 & 0.832 \\
Religion & 0.277 & 0.091 \\
Educational level & 0.052 & 0.697 \\
Monthly family income & 0.092 & 0.802 \\
\hline
\end{tabular}

*p value

The dimension analysis of the PESPC's adapted version was performed through factor analysis for the two domains, expectations and satisfaction. In the expectations domain, all the factor loads were grouped and were above 0.30 . In regard to communality values, most were above 0.52 ; the highest communality was identified for item $6(0.73)$ and the lowest for item 1 (0.36). Some dispersed factor loads were identified for the satisfaction domain (items 13,17,25,29,31,39,40,41). The highest communality value was 0.76 (items 20 and 22 ), and the lowest was 0.27 (item 40).

Two subscales of the satisfaction domain of the PESPC's adapted version (information and provider care) were used to verify the instrument's convergent construct validity along with a subscale of the PSI instrument (trust). Pearson's linear correlation method was used to obtain the results. The results revealed correlation only for the subscale information $(r=0.193$; $p=0.036$ ), while the following values were found for the subscale provider care $(r=-0.005 ; p=0.0554)$.

The reliability of the PESPC's adapted version, verified through stability of measurement (test-retest) for the expectations domain, presented a positive correlation and strong magnitude $(r=0.82 ; p<0.001)$ and presented a positive correlation of moderate magnitude $(r=0.66 ; p<0.001)$ for the satisfaction domain. Reliability was also verified through internal consistency (Cronbach's alpha) for the two domains (expectations and satisfaction) and the following values were obtained, respectively: 0.70 and 0.80 . Two subscales in the expectations domain presented values below 0.70 : provider continuity $(0.29)$ and other services $(0.56)$. All the subscales in the satisfaction domain presented alpha values above 0.70 ; the highest Cronbach's alpha value was obtained by the information subscale (0.87). It is worth noting that the subscale system characteristics in the satisfaction domain obtained Cronbach's alpha 0.87 , above the value obtained in the original version. These results are presented in Table 3.

Table 3 - Cronbach's alpha coefficients of the PESPC's adapted version and the original version by Omar, Schiffman and Bingham (2001). Ribeirão Preto, SP, Brazil 2012

\begin{tabular}{lcc}
\hline \multicolumn{1}{c}{ Factor } & $\begin{array}{c}\text { PESPC's adapted } \\
\text { version }\end{array}$ & $\begin{array}{c}\text { PESPC's original } \\
\text { version }\end{array}$ \\
\hline Expectations & 0.70 & 0.72 \\
Complete care & 0.82 & 0.61 \\
Provider continuity & 0.29 & 0.75 \\
Personalized care & 0.61 & 0.65 \\
Other services & 0.56 & 0.80 \\
Satisfaction & 0.80 & 0.94 \\
Information & 0.89 & 0.91 \\
Provider care & 0.75 & 0.90 \\
Staff interest & 0.76 & 0.93 \\
System characteristics & 0.87 & 0.82 \\
\hline
\end{tabular}

\section{Discussion}

This methodological study sought to adapt and then test the psychometric properties of the adapted version of the Patient Expectations and Satisfaction with Prenatal Care (PESPC) instrument with pregnant women receiving prenatal care in Brazil. The interest in conducting this study emerged from the literature that addresses the assessment of patient satisfaction, which shows that this type of assessment supports the management and implementation of actions, the identification of areas that can be improved to meet the needs of the population, and enable the improvement of elements that potentially generate dissatisfaction.

Additionally, the literature evidences a lack of valid and reliable instruments designed to measure the satisfaction and expectations of pregnant women in relation to prenatal care $^{(9)}$, which also motivated this study. Similar to the original version's study(9), this study's participants originated from heterogeneous groups in terms of age, marital status, socioeconomic status, number of children and gestation period.

In regard to the face and content validity of the PESPC's adapted version performed by an Expert Committee, the instrument's items were considered to be appropriate to assess the expectations and satisfaction of patients in relation to prenatal care. 
Descriptive analysis of the expectations domain revealed evidence of low expectations regarding the subscales complete care and personalized care, as indicated by the corresponding averages, medians, and maximum values. In turn, for the satisfaction domain, averages and medians were close to the interval's lower bound, indicating high satisfaction in the studied sample.

In regard to the divergent construct validity verified among groups for the expectations domain, some hypotheses previously established were confirmed by the statistically significant results, showing there is a relationship between expectations for prenatal care and the following variables: number of children, educational level, and occupation. Similar to the satisfaction domain, the statistically significant results confirmed that the variables gestation period and marital status are related to provider care.

The analysis of convergent construct validity showed significant correlations of some items of one subscale in the PESPC's adapted version with some items in one subscale of the PSI, confirming its validity. Construct validity verified through factor analysis and communality showed strong correlation with factors in the expectations domain, evidenced by the grouping of factor loads. A moderate correlation, identified by the presence of dispersed loads, was observed for the satisfaction domain.

The reliability of the adapted PESPC, verified through internal consistency (Cronbach's alpha), for the instrument's two domains (expectations and satisfaction) resulted in appropriate values ( 0.70 and 0.80 , respectively), similar to the original version $(0.72$ and 0.94 , respectively). These results show the reliability of the adapted version.

It is worth noting that the very low alpha values obtained by the two subscales in the expectations domain (provider continuity and other services) may be explained by the reduced number of items in these scales. The highest value of internal consistency in the satisfaction domain occurred in the subscale system characteristics, 0.87 compared to 0.82 obtained by the original version. All the alpha values were above 0.75 , which shows good internal consistency and consequently satisfactory results.

\section{Conclusion and Final Considerations}

This study's results reveal strong and moderate evidence of validity and reliability of the PESPC version adapted to Brazilian Portuguese when applied among pregnant women receiving prenatal care in the city of Ribeirão Preto, SP, Brazil.

Therefore, the availability of this instrument in Brazil will fulfill a lack of instruments assessing the expectations and satisfaction of pregnant women in relation to prenatal care provided in the country. However, since Brazil is so highly diverse culturally, future studies are suggested to test the adapted version of the Patient Expectations and Satisfaction with Prenatal Care (PESPC) in distinct population samples.

\section{Acknowledgments}

We express thanks for the contribution of professionals and patients who participated in this study and the Coordination for the Improvement of Higher Education Personnel (CAPES).

\section{References}

1. Gill L, White LA. A critical review of patient satisfaction. Leadership Health Serv.2009;22(1):8-19.

2. Linder-Pelz S. Toward a Theory of Patient Satisfaction. Soc Sci Med. 1982;16(5):577-82.

3. Roberts RO, Yawn BP, Wickes SL, Field CS, Garretson M, Jacobsen SJ. Barriers to prenatal care: factors associated with late initiation of care in a middle-class midwestern community. J Fam Pract.1998;47(1):53-61. 4. Dye TD, Wojtowycz MA. Organisational variation, satisfaction and women's time investment in prenatal care. Paediatr Perinatal Epidemiol.1999;13(2):158-69.

5. Linder-Pelz S, Struening EL. The muldimensionality of patient satisfaction with a clinic visit. J Community Health. 1985;10(1):42-54.

6. Van Campen C, Sixma H, Friele RD, Kerbsens JJ, Peters L. Quality of care and patient satisfaction: a review of measuring instruments. Med Care Res Rev. 1995;52(1):109-33.

7. The Health Boards Executive (Hebe) [Internet]. Measurement of patient satisfaction guidelines: health strategy implementation project 2003. [acesso 1 ju 2012]. Tullamore: Health Boards Executive Unit 4; 2003. Disponível em: http://hdl.handle.net/10147/43559

8. Pascoe GC. Patient Satisfaction in Primary Health Care: A literature review and analysis. Eval Program Plann. 1983;6(3-4):185-210.

9. Omar MA, Schiffman RF, Bingham CR. Development and Testing of the Patient Expectations and Satisfaction with Prenatal Care Instrument. Res Nurs Health. 2001;24(3):218-29. 
10. O'Hearn. Deaf women's experiences and satisfaction with prenatal care: a comparative study. Fam Med. 2006;38(10):712-6.

11. Orrantia E, Poole H, Strike J, Zelek, B. Evaluation of a novel model for rural obstetric care. Can J Rural Med. 2010;15(1):14-8.

12. Oliveira AML. Satisfação do Paciente com os cuidados de enfermagem: comparação entre dois hospitais. Rev Esc Enferm USP. 2006;40(1):71-7.

13. Hinshaw AS, Atwood JR. A patient satisfaction instrument: precision by replication. Nurs Res. 1982;31(3):170-5.

14. Ferrer M, Alonso J, Prieto L, Plaza V, Monsó E, Marrades $\mathrm{R}$ et al. Validity and reability of the St George's respiratory Questionnaire after adaptation to a different language and culture: the Spanish example. Euro Respir J. $1996 ; 9(6): 1160-6$.

15. Carvalho ARS, Dantas RAS, Pelegrino FM, Corbi ISA. Adaptação e validação de uma medida de adesão à terapia de anticoagulação oral. Rev. Latino-Am. Enfermagem. 2010;18(3):301-8.

16. Sapnas KG, Zeller RA. Minimizing sample size when using exploratory factor analysis for measurement. J Nurs Meas. 2002;10(2):135-54.

17. Terwee CB, Bot SDM, Boer MR, Windt DAW, Knol $D L$, Dekker J, et al. Quality criteria were proposed for measurement properties of health status questionnaires. J Clin Epidemiol. 2007;60(1):34-42.

18. Keszei Ap, Novak M, Streiner DL. Introduction to health measurement scales. J Psychosom Res. 2010;68(4):319-23.

19. Nunnally JC, Bernstein IH. Psychometric theory. 3rd ed. New York: McGraw-Hill; 1994. 